\title{
ON THE NOTION OF RESIDUAL FINITENESS FOR $G$-SPACES
}

\author{
GOUTAM MUKHERJEE and ANIRUDDHA C. NAOLEKAR
}

(Received 23 February 2001; revised 25 September 2001)

Communicated by S. Gadde

\begin{abstract}
We define equivariant completion of a $G$-complex and define residually finite $G$-spaces. We show that the group of $G$-homotopy classes of $G$-homotopy self equivalences of a finite, residually finite $G$-complex, is residually finite. This generalizes some results of Roitberg.
\end{abstract}

2000 Mathematics subject classification: primary 55P60, $57 \mathrm{~S} 17$.

Keywords and phrases: equivariant completion, residually finite $G$-spaces, residually finite groups, Hopfian groups.

\section{Introduction}

The notion of profinite completion in group theory is well understood and it is well known that profinite completion of a group is residually finite. The notion of profinite completion of Sullivan [8] in homotopy theory motivated Roitberg to introduce the notion of residual finiteness in the homotopy category [7]. He showed that the profinite completion of a path connected CW-complex is residually finite [7, Theorem 1 (a)]. He further showed that for a finife $\mathrm{CW}$-complex $X$ which is residually finite, $\mathscr{E}(X)$, the pointed homotopy classes of self homotopy equivalences is residually finite $[7$, Theorem 3]. This is the homotopy theoretic analogue of the well-known result of Baumslag that the automorphism group of a finitely generated residually finite group is residually finite. The aim of this paper is to prove equivariant versions of the above results of Roitberg.

Let $G$ be a finite group and $G \mathscr{H}$ denote the category of $G$-path connected $G$ $\mathrm{CW}$-complexes (which we abbreviate to $G$-complexes) with base point. All maps and homotopies are based. Following Sullivan, we define the profinite completion 
$\hat{X}_{G}$ of a $G$-complex $X$ (for equivariant completion, generalizing the non equivariant completion of Bousfield-Kan, see [3]). We also introduce the notion of residual finiteness for $G$-spaces and show that for any $X \in G \mathscr{H}$, the profinite completion $\hat{X}_{G}$ is residually finite. Let $\mathscr{E}_{G}(X)$ denote the group of $G$-homotopy classes of equivariant homotopy self equivalences of $X$. One of the main results of the paper is

THEOREM 1.1. Let $X \in G \mathscr{H}$ be finite. Assume that $X$ is residually finite. Then $\mathscr{E}_{G}(X)$ is a residually finite group.

Recall that a theorem of Sullivan [9] and Wilkerson [11] says that if $X$ is a nilpotent finite complex, then $\mathscr{E}(X)$ is commensurable with an arithmetic group and hence, is finitely presented. Thus if $X$ is a finite, nilpotent complex which is also residually finite, then $\mathscr{E}(X)$ being residually finite and finitely presented, is Hopfian. The equivariant analogue of the Sullivan-Wilkerson theorem is proved in [10]. We use this to prove

THEOREM 1.2. If $X \in G \mathscr{H}$ is finite and nilpotent, then $\mathscr{E}_{G}(X)$ is Hopfian.

Convention Throughout, $G$ will denote a finite group and all spaces, maps and homotopies are based and ' $X \in G \mathscr{H}$ is finite' is meant that $X$ is a finite $G$-CWcomplex.

\section{Equivariant completion and residual finiteness}

Recall that a space $F$ is totally finite if the homotopy groups $\pi_{n}(F), n \geq 1$ are finite and if in addition there exists a positive integer $n_{0}$ such that $\pi_{n}(F)=0$ for $n>n_{0}$. A space is of finite type if all its homotopy groups are finitely generated.

A $G$-space $X$ is totally finite if for every subgroup $H$ of $G$, the $H$ fixed point set $X^{H}$ is totally finite.

DEFinITION 2.1. A $G$-space $X$ is residually finite if for any finite $G$-complex $W$ and $\alpha, \beta \in[W, X]_{G}, \alpha \neq \beta$ there exists a $G$-map $f: X \rightarrow Z$ with $Z$ totally finite such that $f_{*}(\alpha) \neq f_{*}(\beta)$ where $f_{*}:[W, X]_{G} \rightarrow[W, Z]_{G}$ is the map induced by $f$.

A $G$-map $f: X \rightarrow Y$ between $G$-spaces is a $\mathbb{F}$-monomorphism if for every finite $W \in G \mathscr{H}$ the induced map $f_{*}:[W, X]_{G} \rightarrow[W, Y]_{G}$ is a monomorphism.

Here is an example of a residually finite space.

EXAMPLE 2.2. Let $X=S^{1} \vee S^{1}$. Then $X$ can be given the structure of a $\mathbb{Z}_{2}$-complex as follows. $X$ has one 0 -cell of the type $\mathbb{Z}_{2} / \mathbb{Z}_{2}$ and one 1-cell of the type $\mathbb{Z}_{2} / e . X$ can then be readily recognized as an equivariant Eilenberg-MacLane space $K(\lambda, 1)$ 
where $\lambda$ is the $O_{\mathbb{Z}_{2}}$-group $\lambda\left(\mathbb{Z}_{2} / e\right)=F_{2}$, the free group of rank two, and $\lambda\left(\mathbb{Z}_{2} / \mathbb{Z}_{2}\right)$ is the trivial group. We claim that $X$ is residually finite as $\mathbb{Z}_{2}$-space. First note that if $W$ is a finite $G$-complex then

$$
[W, K(\lambda, 1)]_{G} \cong \operatorname{Hom}_{O_{G}}\left(\underline{\pi}_{1}(W), \lambda\right) .
$$

(This is true more generally [6]). Now let $\alpha, \beta \in[W, K(\lambda, 1)]_{G}$ be such that $\alpha \neq \beta$. Then clearly $\alpha\left(\mathbb{Z}_{2} / e\right) \neq \beta\left(\mathbb{Z}_{2} / e\right): \pi_{1}\left(W^{e}\right) \rightarrow \lambda\left(\mathbb{Z}_{2} / e\right)$. Since $F_{2}$ is residually finite there exists a finite group $F$ and a homomorphism $\mu: \lambda\left(\mathbb{Z}_{2} / e\right) \rightarrow F$ such that

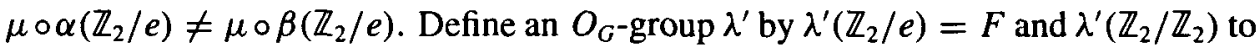
be the trivial group. Then, the map $\mu: \lambda \rightarrow \lambda^{\prime}$ defined by $\mu\left(\mathbb{Z}_{2} / e\right)=\mu$ and $\mu\left(\mathbb{Z}_{2} / \mathbb{Z}_{2}\right)$ being the trivial homomorphism, defines a natural transformation. This gives rise to a $G$-map $h: K(\lambda, 1) \rightarrow K\left(\lambda^{\prime}, 1\right)$ of equivariant Eilenberg-MacLane spaces. Clearly $h_{*}(\alpha) \neq h_{*}(\beta)$. Observe that $K\left(\lambda^{\prime}, 1\right)$ is totally finite. Note that $X$ is not nilpotent as a $\mathbb{Z}_{2}$-space (compare Proposition 2.9).

PROPOSITION 2.3. If $X$ is residually finite as a $G$-space, then $X^{G}$ is residually finite.

ProOF. Let $\alpha, \beta \in\left[W, X^{G}\right], \alpha \neq \beta$ with $W$ a finite $C W$-complex. Then endowing $W$ with the trivial $G$-action, $\alpha, \beta$ can be considered as elements of $[W, X]_{G}$ and it is easy to see that $\alpha \neq \beta$, as elements of $[W, X]_{G}$. Hence there is a totally finite $G$-space $Z$ and a $G$-map $f: X \rightarrow Z$, such that, $f_{*}(\alpha) \neq f_{*}(\beta)$. Then, it follows that $f_{*}^{G}(\alpha) \neq f^{G}(\beta)$.

We can now construct a $G$-space $X$ which is residually finite, if one forgets the group action but is not residually finite when considered as a $G$-space.

EXAMPLE 2.4. Let $G=\mathbb{Z}_{2}$. Let $f: \mathbb{Q} \rightarrow \mathbb{Z}$ denote the only homomorphism between the additive group of rationals and the integers. This map is then realized as a map $f: K(\mathbb{Q}, 1) \rightarrow S^{1}$ of Eilenberg-MacLane spaces. Consider the $O_{G}$-space $T$, defined by, $T(G / G)=K(\mathbb{Q}, 1)$ and $T(G / e)=S^{1}$, with all structure maps as the identity, except the map $T(\hat{\mathfrak{e}}): T(G / G) \rightarrow T(G / e)$, which equals $f$. Then, by the Elmendorf construction [2], there exists a $G$-space $C T$, such that, $C T$ has the homotopy type of $S^{1}$, whereas $C T^{G}$ has the homotopy type of $K(\mathbb{Q}, 1)$. Corollary 1 of [7] shows that $C T^{G}$ is not residually finite, but the underlying space of the $G$-space $C T$, is clearly residually finite. It follows from the above proposition that, $C T$ is not residually finite, as a $G$-space.

We now turn to the definition of equivariant completion. Recall [4, Theorem 3.1, page 134] that, a contravariant functor from $G \mathscr{H}$ to the category of sets, is representable, if and only if, it satisfies the Brown's axioms (the wedge and the MayerVietoris axioms). A functor satisfying the wedge and the Mayer-Vietoris axioms will 
be called a Brownian functor. A compact Brownian functor is a Brownian functor taking values in compact Hausdorff spaces.

We shall need the following two properties of compact Brownian functors.

(1) Suppose $k^{\prime}$ is a contravariant functor defined on the subcategory of $G \mathscr{H}$ consisting of finite $G$-complexes taking values in compact Hausdorff spaces. Suppose that $k^{\prime}$ satisfies the Brown's axioms, whenever they make sense. Then, there is a unique extension of $k^{\prime}$ to a compact Brownian functor $k$, defined by, $k(X)=\operatorname{inv} \lim _{\alpha} k^{\prime}\left(X_{\alpha}\right)$, where the inverse limit is over the finite $G$-subcomplexes $X_{\alpha}$ of $X$.

(2) The arbitrary inverse limit of compact Brownian functors, over a small filtering category, is a compact Brownian functor.

The proofs of both these facts are analogous to the nonequivariant case [8, page 36] and are therefore omitted. We shall use the above properties of compact Brownian functors to introduce equivariant completion as follows.

Step 1 For $X \in G \mathscr{H}$, let $\mathscr{F}_{X}$ denote the category whose objects are $G$-maps $X \rightarrow F$ with $F$ a totally finite $G$-space and morphisms are homotopy commutative diagrams.

LEMMA 2.5. $\mathscr{F}_{X}$ is a small filtering category.

Proof. Recall ([8]) that, to show that the category $\mathscr{F}_{X}$ is small filtering we need to check the smallness, the directedness of $\mathscr{F}_{X}$ and the essential uniqueness of maps in $\mathscr{F}_{X}$. The first condition is clear since we can replace $\mathscr{F}_{X}$ by an equivalent small category, by picking a representative from each $G$-homotopy type of $F$ 's. The second property is also clear as given objects $f_{1}: X \rightarrow F_{1}$ and $f_{2}: X \rightarrow F_{2}$ in $\mathscr{F}_{X}$ we can imbed them in $f_{1} \times f_{2}: X \rightarrow F_{1} \times F_{2}$. The essential uniqueness of maps in $\mathscr{F}_{X}$ follows from the co-equalizer construction in equivariant homotopy theory, which is given by a suitable pushout diagram [4, page 39]. Explicitly, for two morphisms from $\pi^{\prime}: X \rightarrow F^{\prime}$ to $\pi: X \rightarrow F$ in $\mathscr{F}_{X}$ given by $G$-maps $f_{1}, f_{2}: F^{\prime} \rightarrow F$, consider the $G$-space

$$
\left\{(p, x) \in F^{I} \times F^{\prime}: p(0)=f_{1}(x), p(1)=f_{2}(x)\right\}
$$

with diagonal action, where the $G$-action on $F^{\prime}$ is induced by the action on $F$. Let $F^{\prime \prime}$ be the component of the above $G$-space containing the base point, the base point being the constant path at the base point of $F$ in the first factor and the base point of $F^{\prime}$ in the second factor. Then, as in the non-equivariant case [4, page 40], we have an exact sequence

$$
\cdots \rightarrow \pi_{i}\left(F^{\prime \prime}\right)^{H} \rightarrow \pi_{i}\left(F^{\prime}\right)^{H} \rightarrow \pi_{i}\left(F^{H}\right) \rightarrow \cdots,
$$

for every subgroup $H$ of $G$. From this exact sequence it follows that $F^{\prime \prime}$ is a totally finite $G$-space. Now, one gets the required co-equalizer by using a $G$-homotopy from $f_{1} \circ \pi^{\prime}$ to $f_{2} \circ \pi^{\prime}$. 
Step 2 Let $Z \in G \mathscr{H}$ be finite and $F$ a totally finite $G$-space. Then by equivariant obstruction theory [1], it is easy to see that, the homotopy set $[Z, F]_{G}$ is finite. This yields a contravariant functor defined on the sub category of $G \mathscr{H}$ consisting of finite $G$-complexes and taking values in compact Hausdorff spaces. A direct verification shows that this functor satisfies the Brown's axioms whenever they make sense. Then by property (1), we get a compact Brownian functor defined by $S_{F}(Y)=\operatorname{inv}_{\lim _{\alpha}}\left[Y_{\alpha}, F\right]_{G}=[Y, F]_{G}$, where the inverse limit is taken over the finite $G$-subcomplexes of $Y$.

From Step 1 and Step 2 we get a functor on $\mathscr{F}_{X}$ which assigns to each object $\pi: X \rightarrow F$, the compact Brownian functor $S_{F}$ obtained as in Step 2. By property (2) of compact Brownian functors inv $\lim _{\mathscr{F}_{X}} S_{F}$ is again a compact Brownian functor, which assigns, to each $Y \in G \mathscr{H}$, the compact Hausdorff space inv $\lim _{\mathscr{F}_{X}}[Y, F]_{G}$. Therefore, by Brown's representation theorem [4, Theorem 3.1, page 134], there exists a space $\hat{X}_{G}$ in $G \mathscr{H}$ such that for every $G$-complex $Y$ there is a bijection

$$
\left[Y, \hat{X}_{G}\right]_{G} \longleftrightarrow \operatorname{inv} \lim _{\mathscr{F}_{X}}[Y, F]_{G} .
$$

DEFINITION 2.6. The space $\hat{X}_{G}$ is called the equivariant profinite completion of $X$.

Clearly, $\hat{X}_{G}$ comes equipped with a $G$-map $i: X \rightarrow \hat{X}_{G}$, which is determined by the objects of $\mathscr{F}_{X}$ and is called the completion map.

We now prove an important property of equivariant completion. First recall that a $G$-space $X$ is nilpotent if every fixed point set is nilpotent. An equivariant Postnikov decomposition for a $G$-space $B$ consists of $G$-maps $\alpha_{n}: B \rightarrow B_{n}$ and $r_{n+1}: B_{n+1} \rightarrow$ $B_{n}, n \geq 0$ such that $B_{0}$ is a point and $\alpha_{n}$ induces an isomorphism $\underline{\pi}_{q}(B) \rightarrow \underline{\pi}_{q}\left(B_{n}\right)$ for $q \leq n, r_{n+1} \alpha_{n+1}=\alpha_{n}$, and $r_{n+1}$ is the $G$-fibration over a $K\left(\pi_{n+1}(B), n+2\right)$ by a map $k^{n+2}: B_{n} \rightarrow K\left(\underline{\pi}_{n+1}(B), n+2\right)$. On passage to $H$-fixed points, a Postnikov system for $B$ gives a Postnikov system for $B^{H}$. Moreover, every nilpotent $G$-space admits a Postnikov decomposition [4, 2].

PROPOSITION 2.7 (Hasse principle). Let $Y \in G \mathscr{H}$ be finite and $B \in G \mathscr{H}$ be a nilpotent space of finite type. If $f, g: Y \rightarrow B$ are $G$-maps such that $i \circ f$ is $G$-homotopic to $i \circ g$, then $f$ is $G$-homotopic to $g$.

PROOF. The proof is by induction over the stages in the equivariant Postnikov system of $B$ and is parallel to the nonequivariant case. Let $K \rightarrow B_{n+1} \rightarrow B_{n}$ be a part of the equivariant Postnikov decomposition of $B$ (see $[4,2])$, where $K=K(\underline{\pi}, n+1)$ and $\underline{\pi}=\underline{\pi}_{n+1}\left(B_{n+1}\right)$. Suppose $f_{n}: Y \rightarrow B_{n}$ and $f_{n+1}: Y \rightarrow B_{n+1}$ are the $G$-maps constructed from $f$. Now consider the $G$-fibration

$$
\operatorname{Map}(Y, K) \rightarrow \operatorname{Map}\left(Y, B_{n+1}\right) \stackrel{r}{\rightarrow} \operatorname{Map}\left(Y, B_{n}\right)
$$


with the obvious action on the function spaces so that

$$
\text { } \operatorname{Map}\left(Y, B_{n+1}\right)^{G}=\operatorname{Map}_{G}\left(Y, B_{n+1}\right) \text {. }
$$

We then have an ordinary fibration

$$
\operatorname{Map}_{G}(Y, K) \rightarrow \operatorname{Map}_{G}\left(Y, B_{n+1}\right) \stackrel{r}{\rightarrow} \operatorname{Map}_{G}\left(Y, B_{n}\right) .
$$

Consider the homotopy exact sequence of the above fibration

$$
\begin{aligned}
\cdots & \rightarrow \pi_{1}\left(\operatorname{Map}_{G}\left(Y, B_{n}\right), f_{n}\right) \stackrel{l}{\rightarrow} \pi_{0}\left(\operatorname{Map}_{G}(Y, K), f_{n+1}\right) \\
& \stackrel{f_{n+1}}{\rightarrow} \pi_{0}\left(\operatorname{Map}_{G}\left(Y, B_{n+1}\right), f_{n+1}\right) \stackrel{r}{\rightarrow} \pi_{0}\left(\operatorname{Map}_{G}\left(Y, B_{n}\right), f_{n}\right) .
\end{aligned}
$$

Note that $\pi_{0}\left(\operatorname{Map}_{G}(Y, K), f_{n+1}\right)=H_{G}^{n+1}(Y ; \pi)$ where $H_{G}^{n+1}(Y ; \pi)$ denotes the Bredon cohomology group with coefficients in the $O_{G}$ group $\underline{\pi}$ [1]. Here $\tilde{f_{n+1}}$ denotes the map given by the action of $H_{G}^{n+1}(Y, \underline{\pi})$ on $\left(f_{n+1}\right) \in \pi_{0}\left(\operatorname{Map}_{G}(Y, K), f_{n+1}\right)$ obtained by equivariant obstruction theory [5]. Clearly, the image $I=I\left(f_{n+1}\right)$ is the isotropy subgroup of the point $\left(f_{n+1}\right)$ and the map $r$ collapses the orbits of the action of $H_{G}^{n+1}(Y, \pi)$. Thus we get an exact sequence

$$
0 \rightarrow I\left(f_{n+1}\right) \rightarrow H_{G}^{n+1}(Y, \underline{\pi}) \rightarrow \operatorname{orbit}\left(f_{n+1}\right) \rightarrow 0 .
$$

We proceed as in the non-equivariant case and repeat the above argument for maps into completions $\widehat{B}_{G}$, to get a ladder whose top row being the above exact sequence, the base row being the exact sequence

$$
0 \rightarrow I\left(\hat{f_{n+1}}\right) \rightarrow H_{G}^{n+1}(Y, \underline{\hat{\pi}}) \rightarrow \operatorname{orbit}\left(\hat{f_{n+1}}\right) \rightarrow 0,
$$

and with induced maps $c_{0}: I\left(f_{n+1}\right) \rightarrow I\left(\hat{f_{n+1}}\right), c: H_{G}^{n+1}(Y, \underline{\pi}) \rightarrow H_{G}^{n+1}(Y, \underline{\hat{\pi}})$ and $c_{1}: \operatorname{orbit}\left(f_{n+1}\right) \rightarrow \operatorname{orbit}\left(\hat{f_{n+1}}\right)$. Here, the $O_{G}$-group $\underline{\hat{\pi}}$ is defined by the group completion $\hat{\hat{\pi}}(G / H)=\widehat{\pi} \widehat{(G / H})$. Also note that by property (1) of compact Brownian functor the map $c: H_{G}^{n+1}(Y, \underline{\pi}) \rightarrow H_{G}^{n+1}(Y, \underline{\hat{\pi}})$, is a finite completion. With this at our disposal the rest of the proof is exactly similar to the non-equivariant case.

Equivariant completion yields, as in the nonequivariant case ([7, Theorem 1]), examples of residually finite spaces.

Proposition 2.8. If $X \in G \mathscr{H}$, then $\hat{X}_{G}$ is residually finite.

Suppose that $f: X \rightarrow Y$ is a $G$-map with $Y$ residually finite. If $f$ is a $\mathbb{F}$ monomorphism, then $X$ is residually finite. The Hasse principle implies that if $X \in G \mathscr{H}$ is nilpotent and of finite type, then the completion map $i: X \rightarrow \hat{X}_{G}$ is a $\mathbb{F}$-monomorphism. Both these facts put together imply

PROPOSITION 2.9. If $X \in G \mathscr{H}$ is nilpotent and of finite type, then $X$ is residually finite. 


\section{Proof of the main theorem}

In this section we prove our main theorem which gives a sufficient condition for the group $\mathscr{E}_{G}(X)$ to be Hopfian. The main step in proving this (as in the non-equivariant case) is showing that, under suitable conditions, the group $\mathscr{E}_{G}(X)$ is residually finite.

DEFINITION 3.1. Let $[f]: X \rightarrow Y$ be a morphism in $G \mathscr{H}$. $f$ is said to represent an epimorphism in $G \mathscr{H}$ if for any two maps $\alpha, \beta: Y \rightarrow Z$ in $G \mathscr{H}, \alpha \circ f$ is $G$-homotopic to $\beta \circ f$ implies $\alpha$ is $G$-homotopic to $\beta$.

Suppose that $X$ and $Y_{0}$ are in $G \mathscr{H}$ and $\left[X, Y_{0}\right]_{G}=\left\{\left[f_{1}\right], \ldots,\left[f_{r}\right]\right\}$. Define $Y=Y_{0} \times \cdots \times Y_{0}$ with $r$ factors. Then $Y$ is a $G$-complex with the diagonal $G$ action. Consider the $G$-map $f: X \rightarrow Y$ by $f=\left(f_{1}, \ldots, f_{r}\right)$. Let $M(Y)$ denote the monoid of equivariant self homotopy equivalences of $Y$ preserving the base point. Each element of the symmetric group $S_{r}$ induces a self map of the $G$-space $Y$ by permuting its coordinates. This gives an embedding of $S_{r}$ into $M(Y)$.

LEMMA 3.2. With the above notation, if $e: X \rightarrow X$ represents an epimorphism in $G \mathscr{H}$, then e determines a unique $\sigma \in S_{r} \subseteq M(T)$ such that $f \circ e$ is $G$-homotopic to $\sigma \circ f$. The assignment $e \mapsto \sigma$ induces a monoid homomorphism $\psi: E(X) \rightarrow S_{r} \subseteq$ $M(T)$, where $E(X)$ is the monoid of equivariant self epimorphisms of the $G$-space $X$.

ProOF OF THEOREM 1.1. Let $\theta \in \mathscr{E}_{G}(X), \theta \neq i d$. We shall exhibit a homomorphism $\eta: \mathscr{E}_{G}(X) \rightarrow F$ with $F$ a finite group such that $\eta(\theta) \neq i d$. Since $X$ is residually finite, we have a map $f: X \rightarrow Y_{0}$ of with $Y_{0}$ totally finite such that $f_{*}(\theta) \neq f_{*}(i d)$. Since $X$ is finite and $Y_{0}^{H}$ is totally finite one observes using equivariant obstruction theory [1] that the equivariant homotopy set $\left[X, Y_{0}\right]_{G}$ is finite. Thus by Lemma 3.2 there is a $r>1$ and a $\sigma \in S_{r} \subseteq M\left(Y_{0}\right)$ such that $f \circ \theta$ is $G$-homotopic to $\sigma \circ f$ and $f_{*}(\theta) \neq f_{*}(i d)$. Hence $\sigma \neq 1$. Now the monoid homomorphism $\psi: E(X) \rightarrow S_{r}$ of Lemma 3.2 restricted to $M(X)$ induces a group homomorphism $\eta: \mathscr{E}_{G}(X) \rightarrow S_{r}$ such that $\eta(\theta) \neq i d$. This completes the proof.

Proof OF THEOREM 1.2. Recall that by Proposition $2.9, X$ is residually finite. Thus $\mathscr{E}_{G}(X)$ is a residually finite group. Moreover it follows from the work of Triantafillou [10, Theorem 1.2] that $\mathscr{E}_{G}(X)$ is commensurable with an arithmetic subgroup of $\mathscr{E}_{G}\left(X_{0}\right)$, where $X_{0}$ is the equivariant rationalisation of $X$. Thus $\mathscr{E}_{G}(X)$ is finitely generated. The theorem now follows as finitely generated residually finite groups are Hopfian. This completes the proof.

There are situations where it is not difficult to recognize the group $\mathscr{E}_{G}(X)$ as being residually finite. 
EXAMPLE 3.3. Let $\lambda$ be an $O_{G}$-group. Let $n \geq 1$. If $n>1$, then $\lambda$ is abelian. Then if $\lambda$ has the property that $\lambda(G / H)$ is finitely generated residually finite group for all subgroups $H$, then it is not difficult to see that $\mathscr{E}_{G}(X)$ is residually finite where $X$ is the equivariant Eilenberg-MacLane space $K(\lambda, n)$.

EXAMPLE 3.4. As another example, suppose that $X \in G \mathscr{H}$ is a finite nilpotent space such that for any $G$-homotopy equivalence $f: X \rightarrow X$ which is not $G$ homotopic to identity, there exists a subgroup $H$ of $G$ such that $f^{H}: X^{H} \rightarrow X^{H}$ is not homotopic to the identity. Then $\mathscr{E}_{G}(X)$ is residually finite (compare Proposition 3.5).

We end with the following

Proposition 3.5. Suppose $X \in G \mathscr{H}$ is a finite and nilpotent. Further assume that for each subgroup $H, K$ of $G$

(1) $\left[X^{K}, X^{H}\right]$ is a group and

(2) $\left[X^{K}, \Omega^{n} X^{H}\right]$ is trivial for $n \geq 1$.

Then $\mathscr{E}_{G}(X)$ is residually finite.

PROOF. First note that for every subgroup $H$ of $G, X^{H}$ is nilpotent of finite type and hence $X^{H}$ is residually finite [7]. Now let $[f] \in \mathscr{E}_{G}(X)$ such that $[f] \neq[i d]$. Then there exists a subgroup $H$ of $G$ such that $\left[f^{H}\right] \neq[i d]$, otherwise, by [2, Theorem 3], the natural family $\left\{\left[f^{H}\right]\right\}$ would correspond to id $: X \rightarrow X$ and this would mean $f \simeq_{G} i d$. The group $\mathscr{E}\left(X^{H}\right)$ is residually finite by [7, Theorem 3]. Using the obvious homomorphism $\mathscr{E}_{G}(X) \rightarrow \mathscr{E}\left(X^{H}\right)$ one sees that the group $\mathscr{E}_{G}(X)$ is also residually finite. This completes the proof.

Corollary 3.6. Suppose $X \in G \mathscr{H}$ is a finite and nilpotent. Moreover suppose that the G-action on $X$ is free outside the base point. Then $\mathscr{E}_{G}(X)$ is residually finite.

EXAMPLE 3.7. Let $X=S^{2} \vee S^{2}$. Then $X$ can be given a $\mathbb{Z}_{2}$-complex structure by interchanging the copies of $S^{2}$. Then $X$ satisfies the hypothesis of the corollary and hence $\mathscr{E}_{G}(X)$ is residually finite. It is easy to see that this group is non-zero.

Acknowledgement We thank Professor P. Sankaran for his valuable comments.

\section{References}

[1] G. E. Bredon, Equivariant cohomology theories, Lecture Notes in Math. 34 (Springer, Berlin, 1967).

[2] A. D. Elmendorf, 'Systems of fixed point sets', Trans. Amer. Math. Soc. 277 (1983), 275-284. 
[3] J. P. May, 'Equivariant completion', Bull. London Math. Soc. 14 (1982), 231-237.

[4] - Equivariant homotopy and cohomology theory, CBMS Regional Conf. Series in Math. 91 (Amer. Math. Soc., Providence, 1996).

[5] A. Mukherjee and G. Mukherjee, 'Bredon-Illman cohomology with local coefficients', Quart. J. Math. Oxford (2) 47 (1996), 199-219.

[6] G. Mukherjee, 'Hopfian and co-Hopfian G-CW-complexes', Proc. Amer. Math. Soc. 125 (1997), 1229-1236.

[7] J. Roitberg, 'Residual finiteness in homotopy theory', J. Pure Appl. Algebra 32 (1984), 347-358.

[8] D. Sullivan, 'Genetics of homotopy theory and the Adams conjecture', Ann. of Math. (2) 100 (1974), 1-79.

[9] _ - 'Infinitesimal computations in topology', Publ. Inst. Haute Etude Sci. 47 (1978), 269-331.

[10] G. Triantafillou, 'An algebraic model for G-homotopy types', Astérisque 113-114 (1984), 312337.

[11] C. W. Wilkerson, 'Applications of minimal simplicial groups', Topology 15 (1976), 111-130.

Stat.-Math. Unit

Indian Statistical Institute

203, B. T. Road

Calcutta 700035

India

e-mail: goutam@isical.ac.in, anirudha@isical.ac.in 
